\title{
Surface roughness and packaging tightness affect calcium lactate crystallization on Cheddar cheese
}

\author{
P. Rajbhandari and P. S. Kindstedt ${ }^{1}$ \\ Department of Nutrition and Food Sciences, University of Vermont, Burlington 05405-0086
}

\begin{abstract}
Calcium lactate crystals that sometimes form on Cheddar cheese surfaces are a significant expense to manufacturers. Researchers have identified several postmanufacture conditions such as storage temperature and packaging tightness that contribute to crystal formation. Anecdotal reports suggest that physical characteristics at the cheese surface, such as roughness, cracks, and irregularities, may also affect crystallization. The aim of this study was to evaluate the combined effects of surface roughness and packaging tightness on crystal formation in smoked Cheddar cheese. Four 20-mm-thick cross-section slices were cut perpendicular to the long axis of a retail block $(\sim 300 \mathrm{~g})$ of smoked Cheddar cheese using a wire cutting device. One cut surface of each slice was lightly etched with a cheese grater to create a rough, grooved surface; the opposite cut surface was left undisturbed (smooth). The 4 slices were vacuum packaged at $1,10,50$, and $90 \mathrm{kPa}$ (very tight, moderately tight, loose, very loose, respectively) and stored at $1^{\circ} \mathrm{C}$. Digital images were taken at 1,4 , and $8 \mathrm{wk}$ following the first appearance of crystals. The area occupied by crystals and number of discrete crystal regions (DCR) were quantified by image analysis. The experiment was conducted in triplicate. Effects of storage time, packaging tightness, surface roughness, and their interactions were evaluated by repeated-measures ANOVA. Surface roughness, packaging tightness, storage time, and their 2-way interactions significantly affected crystal area and DCR number. Extremely heavy crystallization occurred on both rough and smooth surfaces when slices were packaged loosely or very loosely and on rough surfaces with moderately tight packaging. In contrast, the combination of rough surface plus very tight packaging resulted in dramatic decreases in crystal area and DCR number. The combination of smooth surface plus very tight packaging virtually eliminated crystal formation, presumably by eliminating available sites for nucleation. Cut-and-wrap operations may
\end{abstract}

Received June 30, 2013.

Accepted December 13, 2013.

${ }^{1}$ Corresponding author: paul.kindstedt@uvm.edu significantly influence the crystallization behavior of Cheddar cheeses that are saturated with respect to calcium lactate and thus predisposed to form crystals. Key words: calcium lactate, crystal, Cheddar cheese

\section{INTRODUCTION}

Calcium lactate (CL) crystals that sometimes form on the surface of Cheddar cheese may pose a significant expense to manufacturers due to consumer rejection of cheeses that prominently display the white crystalline deposits. Not surprisingly, the chemical composition of Cheddar cheese strongly influences the likelihood of CL surface crystallization: higher contents of total protein, total and soluble calcium, and lactic acid (Agarwal et al., 2005, 2006a, 2008) and lower salt-in-moisture and cheese pH levels (Swearingen et al., 2004; Agarwal et al., 2008) have all been shown to be associated with higher crystal incidence. Cheddar cheese that is smoked naturally is particularly susceptible to CL crystallization because of chemical changes that occur during the smoking process; namely, surface dehydration and reduction in surface $\mathrm{pH}$, which elevate calcium and lactate ion concentrations in the serum phase at the cheese surface (Rajbhandari et al., 2009). The strong tendency of naturally smoked Cheddar to form CL crystals renders it a useful model for studying CL crystallization behavior and the various postmanufacturing factors that influence CL crystal formation (Rajbhandari and Kindstedt, 2005b, 2008; Rajbhandari et al., 2013).

Cheese microflora also play an important role, including the specific starter culture strains used in cheese making and their salt tolerance (Swearingen et al., 2004; Agarwal et al., 2008), as well as the levels of nonstarter bacteria able to convert $\mathrm{L}(+)$ lactic acid to the less soluble racemic mixture of $\mathrm{L}(+)$ and $\mathrm{D}(-)$ lactic acid (Johnson et al., 1990b; Somers et al., 2001; Chou et al., 2003; Agarwal et al., 2005, 2006b). Postmanufacturing parameters such as storage temperature, packaging with a gas flush, and tightness of the packaging film have also been shown to influence crystallization in complex ways. For example, storage at lower temperatures may increase the incidence of surface crystals due to lower CL solubility (Johnson et al., 1990a; Rajbhandari et 
al., 2013). However, storage at higher temperature may also induce greater crystallization (especially if followed by a period of low temperature storage) as a result of increased growth of racemase-positive nonstarter bacteria that convert $\mathrm{L}(+)$ to $\mathrm{D}(-)$ lactic acid, and increased incidence of free serum release at the cheese surface; that is, weeping or sweating (Johnson et al., 1990a,b; Chou et al., 2003; Rajbhandari et al., 2013). Packaging with a carbon dioxide gas flush may also increase surface crystallization when carbon dioxide from the flush dissolves into the serum phase at the cheese surface to produce carbonic acid, thereby lowering the $\mathrm{pH}$ and increasing the level of soluble calcium (Dybing et al., 1988; Agarwal et al., 2005). More importantly, the presence of headspace between the cheese surface and packaging film, which is often a feature of gas-flushed packaging, strongly promotes CL surface crystals, even when flushing is performed with nitrogen gas in place of carbon dioxide (Agarwal et al., 2005). Furthermore, the loosening over time of an initially tight gas-flushed packaging film has also been shown to promote CL surface crystal formation on Cheddar (Johnson et al., 1990a).

Indeed, packaging tightness (or looseness/headspace presence) seems to be a major factor in CL crystallization irrespective of whether Cheddar cheese is gas flushed or vacuum packaged (Johnson et al., 1990a,b; Agarwal et al., 2005; Valentine et al., 2007), which suggests that surface characteristics influence crystal formation by creating sites that are favorable for nucleation and growth. Nucleation is a critically important step for controlling crystallization in foods (Hartel, 2001), and nucleation at the surface of nonfood materials (e.g., glass) is strongly dependent on surface topography and characteristics such as tips, cracks and scratches, foreign particles, and surrounding atmosphere (Zanotto and Fokin, 2003). With respect to Cheddar cheese, Johnson (2004) postulated that uneven or rough surfaces created by cutting machines or physical handling may serve as nucleation sites, thereby increasing the propensity for CL crystallization when such cheese is packaged loosely, and especially when free serum forms at the surface. However, information about the effect of surface features on CL crystal formation on Cheddar cheese appears to be mostly anecdotal in nature, and the effects of cheese surface roughness, packaging tightness, and their possible interaction on CL crystallization have not been studied systematically.

Consistent with Johnson (2004), we hypothesize that the occurrence of surface irregularities, such as grooves etched into the cheese surface during cutting, combined with loose packaging create sites that are suitable for crystal nucleation and growth and increase the likelihood of CL crystal defects in Cheddar cheese.
Furthermore, we hypothesize that suppression of these sites could reduce or eliminate surface crystal incidence. Previously, we used image analysis techniques to measure total crystal area and discrete crystal region (DCR) number on Cheddar cheese, the latter serving as an indicator of the number of surface sites where nucleation occurred (Rajbhandari and Kindstedt, 2005b, 2008; Rajbhandari et al., 2013). The present study similarly used image analysis to evaluate the effects of surface roughness and packaging tightness on CL surface crystallization in smoked Cheddar cheese.

\section{MATERIALS AND METHODS}

Three retail samples of smoked Cheddar cheese were obtained from a local supermarket. Product code information supplied by the manufacturer was used to confirm that the 3 samples were produced from the same vat on the same day. These cheeses were produced by a milled curd procedure using an automated production line that included horizontal enclosed vats (22,727-kg capacity) for coagulating the milk and cooking the curd; an enclosed conveyor series for continuous dewheying, cheddaring, and milling; enclosed mechanical metering of dry salt and automated stirring of salted curd; and block-forming towers that produced 19.1-kg blocks. Pasteurized whole milk, not standardized for fat content, was inoculated with mesophilic lactic bulk starter prepared with external $\mathrm{pH}$ control, and the milk was coagulated with fermentation-produced chymosin. Following cutting, the vat was cooked to $38.3^{\circ} \mathrm{C}$ and drained at approximately $\mathrm{pH}$ 6.2. The cheddared curd was milled and salted at $\mathrm{pH} 5.7$, with the time from renneting to salting spanning approximately 3 $\mathrm{h}$. The blocks were vacuum packaged and aged at the cheese plant for $3 \mathrm{mo}$ at $12.8^{\circ} \mathrm{C}$ before being cut into retail-sized chunks $(\sim 60 \times 45 \times 100 \mathrm{~mm})$ for smoking according to the cheese manufacturer's standard protocol. Cheeses were smoked at a commercial smokehouse where they were exposed to natural vaporous smoke at $20^{\circ} \mathrm{C}$ for approximately $6 \mathrm{~h}$. After smoking, the samples were vacuum packaged and distributed to retail stores.

The cheese samples were approximately 9 mo of age from the date of manufacture when they were purchased at a retail outlet for this study. Each sample, which formed the basis for 1 experimental replication, was cut into 4 cross-sectional $(\sim 60 \times 45 \times 20 \mathrm{~mm})$ slices using a wire-cutting device according to the plan shown in Figure 1. The 2 opposite newly cut cross-sectional surfaces $(60 \times 45 \mathrm{~mm})$ of each slice, corresponding to the interior of the cheese sample, served as the experimental units. For each slice, one of the cross-sectional surfaces was lightly etched using a cheese grater to create an irregular grooved surface (rough surface). The 
opposite surface was left undisturbed (smooth surface). The 4 slices, each containing one rough and one smooth cross-sectional surface, were then randomly assigned to 4 different packaging tightness treatments: very tight (1 $\mathrm{kPa}$ : complete contact between the packaging film and cheese surface; strong pressure exerted by the packaging film on the cheese surface); moderately tight (10 kPa: almost complete contact between the packaging film and cheese surface except around the edges of the slice; moderate pressure exerted by the packaging film on the cheese surface); loose (50 kPa: limited contact between the packaging film and cheese surface but very limited headspace; negligible pressure exerted by the packaging film on the cheese surface); and very loose (90 kPa: almost no contact between the packaging film and cheese surface and considerable headspace; negligible pressure exerted by the packaging film on the cheese surface). The 4 packaging tightness levels were chosen to encompass the approximate tightness conditions that occur commonly in retail-packaged Cheddar cheeses, ranging from extremely loose gas-flushed packaged shredded and cubed cheeses with substantial headspace to very tightly vacuum packaged cheese chunks. Gas flushing was not performed on any treatment because our objective was to investigate the effects of surface roughness and packaging tightness independent of potential confounding effects of gas flushing. Samples were packaged in 3.2-mil polyethylene-polyamide laminated pouches (Industrial Paper and Packaging Inc., Middleton, WI), using a Multivac vacuum packaging machine (model A300/16, Multivac Sepp Haggenmuller KG, Wolfertschwenden, Germany). The vacuum packaged slices were hung vertically in a laboratory incubator (model 146E Low Temperature Incubator, Fisher Scientific, Pittsburgh, PA) maintained at $1^{\circ} \mathrm{C}$ and stored for 20 wk. The storage temperature of $1^{\circ} \mathrm{C}$ was chosen to accelerate crystallization due to low CL solubility and to prevent mold growth in the moderately and very loosely packaged treatments, which were not gas flushed and therefore vulnerable to potential mold growth.

Digital photographs of the rough and smooth surfaces were taken with the packaging film intact at 1,4 , and 8

$(100 \times 60 \times 45 \mathrm{~mm})$

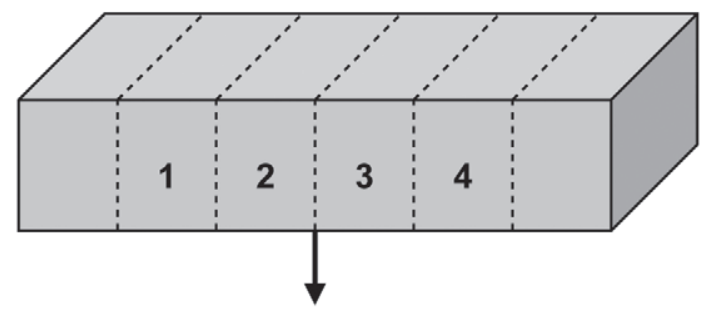

$(60 \times 45 \times 20 \mathrm{~mm})$

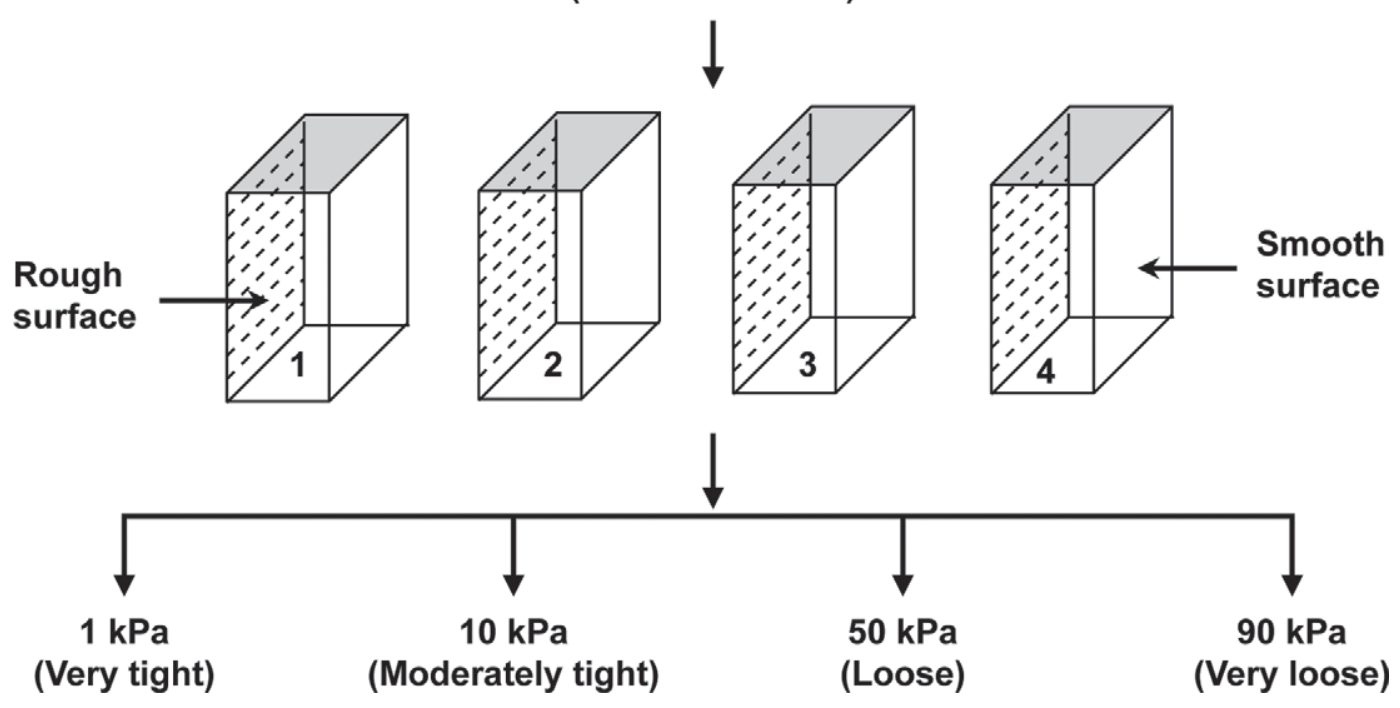

Figure 1. Schematic representation of sectioning plan used to prepare 4 experimental cross-sectional slices from smoked Cheddar cheese $(\sim 300 \mathrm{~g})$ samples. For each experimental slice, one cut surface was etched lightly with a cheese grater to create a rough surface; the opposite surface was left undisturbed (smooth surface). The 4 slices were then randomly assigned to 4 packaging treatments: very tight (1 kPa), moderately tight $(10 \mathrm{kPa})$, loose $(50 \mathrm{kPa})$, and very loose $(90 \mathrm{kPa})$. 
wk following the first appearance of crystals using a still digital camera (model no. E5200, 5.1 megapixel, Nikon Corp., Tokyo, Japan). The entire study was conducted in triplicate using the 3 cheese samples as experimental replications. Images of rough and smooth surfaces were analyzed for (1) percentage of total cheese surface area occupied by crystals, and (2) number of DCR expressed per $\mathrm{cm}^{2}$ of cheese surface area using image analysis software (Metamorph offline, version 7.1.2.0, Molecular Devices LLC, Sunnyvale, CA) as described by Rajbhandari and Kindstedt (2005b, 2008). The data obtained from image analysis were analyzed by ANOVA according to a repeated-measures design with 2 within-subject variables and 1 between-subject variable (SPSS software version 15.0, SPSS Inc., Chicago, IL). The within-subject variables were surface characteristic (2 levels: rough and smooth) and storage time (3 levels: 1, 4, and $8 \mathrm{wk}$ ); the between-subject variable was packaging tightness (2 levels: moderately tight and very tight). Data from the loose and very loose packaging treatments were not analyzed statistically for the reasons described below. Significance was determined at the 0.05 level of probability.

Slices that were packaged moderately tightly and very tightly were held at $1^{\circ} \mathrm{C}$ for an additional $12 \mathrm{mo}$ after completion of the 20-wk study for observation, at which time additional photographs were taken. After this, the tightly packaged samples were finely grated and combined to form a composite sample for chemical analyses, conducted in duplicate. Moisture content was determined by drying in a forced-draft oven (model 130DM, Thermo Electron, Winchester, VA) at $100^{\circ} \mathrm{C}$ for $24 \mathrm{~h}$. Fat content was determined by Babcock method (Marshall, 1993). Total protein was determined by the Kjeldahl method, using a semi-micro block digestion method (IDF, 1993), and salt content was determined using a chloride analyzer (M926, Nelson and Jameson, Marshfield, WI).

\section{RESULTS AND DISCUSSION}

Naturally smoked Cheddar cheese is prone to CL crystallization because dehydration occurs during smoking, which concentrates calcium and lactate ions in the serum phase of the cheese. Furthermore, volatile acidic smoke components are absorbed into the serum phase, which lowers the cheese $\mathrm{pH}$ and increases soluble calcium levels (Rajbhandari et al., 2009). The propensity of smoked Cheddar to crystallize renders it a useful model in the study of CL crystallization (Rajbhandari and Kindstedt, 2005a,b, 2008; Rajbhandari et al., 2013). The specific cheese samples chosen for the present study already displayed slight surface crystallization at the time of receipt, thereby demonstrating that the cheeses were saturated with respect to calcium and lactate ions and thus predisposed to form CL crystals.

The composite cheese sample contained $30.92 \%$ moisture, $37.75 \%$ fat, $26.83 \%$ total protein, and $1.98 \%$ salt. Moisture content was lower than observed previously in smoked Cheddar produced at the same cheese plant under similar conditions (Rajbhandari and Kindstedt, 2005a; Rajbhandari et al., 2009). This was probably caused by evaporative moisture loss from the specimens over the course of the study because of their large surface area-to-volume ratio combined with the long duration of storage $(72 \mathrm{wk})$ that preceded composition testing.

Visible crystals were first detected during wk 6 of storage at $1^{\circ} \mathrm{C}$ on both rough and smooth surfaces when cheese slices were packaged either loosely or very loosely. These loose packaging treatments were chosen to represent tightness levels commonly observed with gas-flushed cubed and shredded Cheddar cheeses. Once initiated, crystal growth on these loosely packaged slices proceeded rapidly and the surfaces developed heavy crystallization during the next $8 \mathrm{wk}$ (data not shown). In contrast, visible crystals did not become evident until wk 12 of storage on slices that were packaged moderately tightly or very tightly (Figures 2 and 3 ). Therefore, the lack of contact between the packaging film and cheese surfaces that occurred when slices were packaged loosely or very loosely appeared to have an overarching effect on promoting CL crystallization, irrespective of the contour of the cheese surface or the amount of headspace between the cheese surface and packaging film. These results are consistent with the findings reported by Johnson et al. (1990a) and Agarwal et al. (2005) and support the view that loose packaging accelerates CL crystallization in cheeses that are already saturated with respect to calcium lactate. Unfortunately, unanticipated sloughing off of crystals from surfaces due to slippage of the vertically hung samples within the packaging film made it impossible to perform reliable image analysis measurements on the slices packaged loosely or very loosely. Therefore, only data from the moderately tight and very tight treatments were analyzed statistically and are presented.

Statistical analyses of the data obtained from the moderately and very tightly packaged slices determined that surface roughness, packaging tightness, storage time, and their 2-way interactions all significantly affected total crystal area and DCR number (Table 1). Graphic representations of total crystal area (Figure 2) and DCR number (Figure 3) during the 20-wk storage period serve to illustrate the nature of the main and interacting effects. For example, it was evident that total crystal area (Figure 2) and DCR number (Figure 3) generally increased during storage once crystallization 


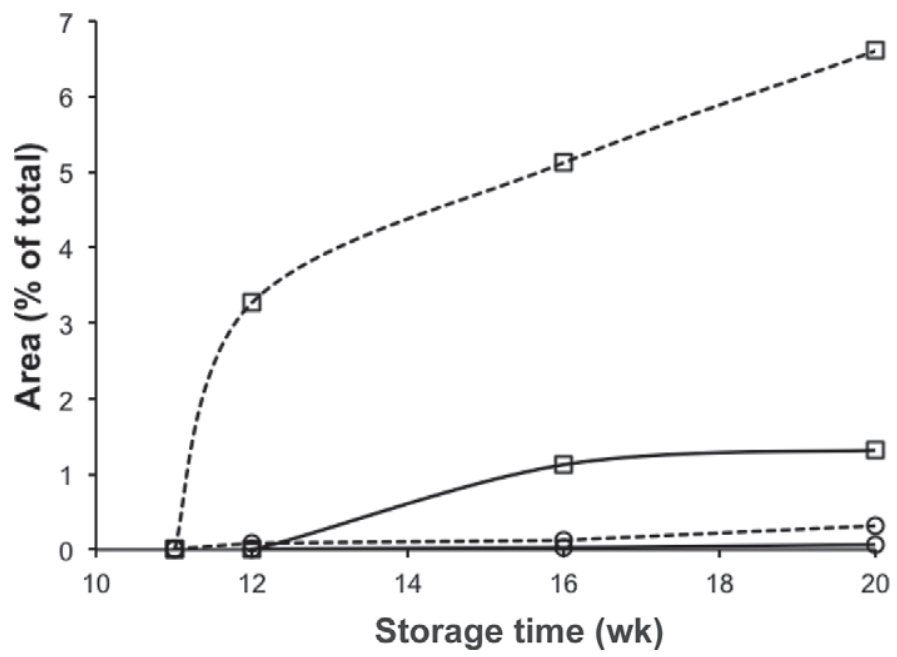

Figure 2. Changes in the total area occupied by crystals, expressed as a percentage of total cheese surface area, on slices of smoked Cheddar cheese that were vacuum packaged very tightly $(\bigcirc)$ or moderately tightly $(\square)$ and stored for $20 \mathrm{wk}$ at $1^{\circ} \mathrm{C}$. Dashed lines correspond to rough surfaces and solid lines to smooth surfaces (see Figure 1).

commenced on wk 12. However, increases were greater for slices packaged moderately tightly than for those packaged very tightly, in keeping with the significant interaction of storage time by packaging tightness (Table 1). Furthermore, rough surfaces displayed greater increases in total crystal area and DCR number than did smooth surfaces at the same packaging tightness, but the effect of roughness was much larger for slices packaged moderately tightly than for those packaged very tightly. Because of these interactions between surface roughness and packaging tightness and between surface roughness and storage time, the combination of a rough cheese surface and moderately tight packaging resulted in disproportionately large numbers of nucleation sites on the surface that gave rise to the development of discrete visible crystals over the next $8 \mathrm{wk}$ of storage (Figure 3), which in turn resulted in

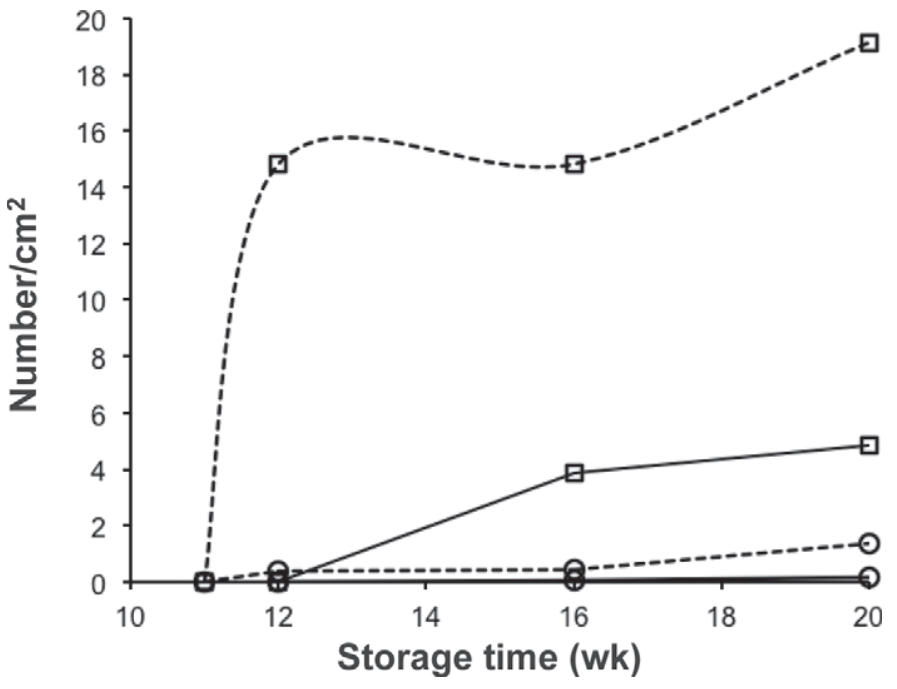

Figure 3. Changes in the number of discrete crystal regions, expressed per $\mathrm{cm}^{2}$ of cheese surface area, on slices of smoked Cheddar cheese that were vacuum packaged very tightly $(\bigcirc)$ or moderately tightly $(\square)$ and stored for $20 \mathrm{wk}$ at $1^{\circ} \mathrm{C}$. Dashed lines correspond to rough surfaces and solid lines to smooth surfaces (see Figure 1).

disproportionately extensive crystal coverage (Figure 2 ). At the other extreme, the combination of smooth surface and very tight packaging virtually prevented the formation of surface crystals during the same period, as evidenced in the measurements of total crystal area (Figure 2) and DCR number (Figure 3).

Photos of cheese slices from one of the experimental replications, taken after $20 \mathrm{wk}$ of storage at $1^{\circ} \mathrm{C}$ (i.e., 8 wk after visible crystals were first observed), are shown in Figure 4. Similar results were obtained for all 3 replications. No visible crystals were present on either the smooth (Figure 4A) or the rough (Figure 4B) surface of the slice that was packaged very tightly. The tightly packaged slices displayed complete contact between the packaging film and cheeses surface, and the surfaces appeared very smooth because of the pressure of the film on the cheese surface. Thus, the downward

Table 1. Degrees of freedom, mean squares (MS), and probabilities for total crystal area and number of discrete crystal regions (DCR) on smoked Cheddar cheese slices containing rough and smooth surfaces that were packaged very tightly or moderately tightly and stored for 8 wk at $1^{\circ} \mathrm{C}$ following the onset of crystallization

\begin{tabular}{|c|c|c|c|c|c|}
\hline \multirow[b]{2}{*}{ Factor } & \multirow[b]{2}{*}{ df } & \multicolumn{2}{|c|}{ Area } & \multicolumn{2}{|c|}{ DCR } \\
\hline & & MS & $P$-value & MS & $P$-value \\
\hline Packaging tightness (PT) & 1 & 70.91 & 0.01 & 759 & $<0.01$ \\
\hline Surface roughness (SR) & 1 & 42.03 & 0.05 & 442 & 0.02 \\
\hline $\mathrm{SR} \times \mathrm{PT}$ & 1 & 36.82 & 0.06 & 363 & 0.03 \\
\hline Storage time (ST) & 2 & 4.69 & $<0.01$ & 20 & 0.02 \\
\hline $\mathrm{ST} \times \mathrm{PT}$ & 2 & 3.70 & $<0.01$ & 12.1 & 0.05 \\
\hline $\mathrm{SR} \times \mathrm{ST}$ & 2 & 0.92 & 0.20 & 4.0 & 0.25 \\
\hline $\mathrm{SR} \times \mathrm{ST} \times \mathrm{PT}$ & 2 & 0.66 & 0.30 & 2.9 & 0.36 \\
\hline
\end{tabular}


A

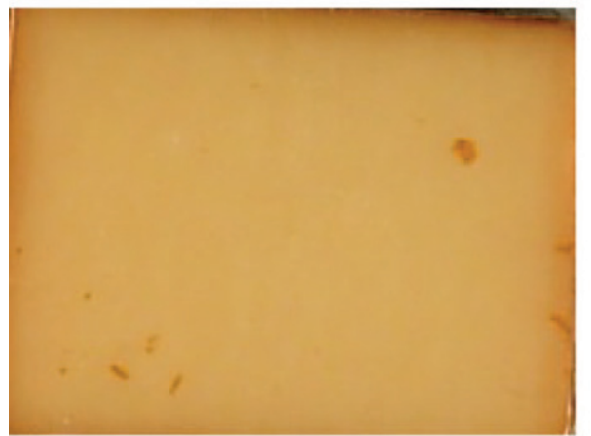

C

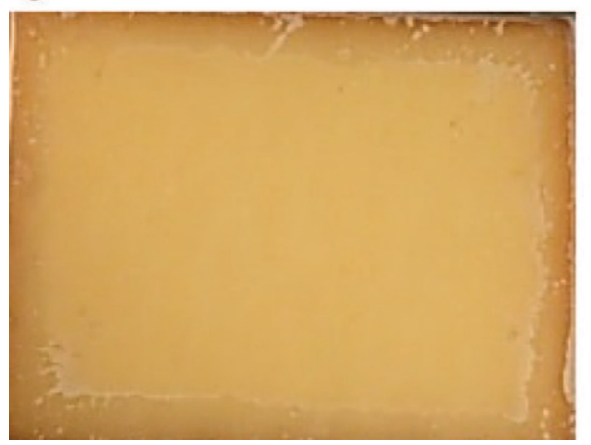

B

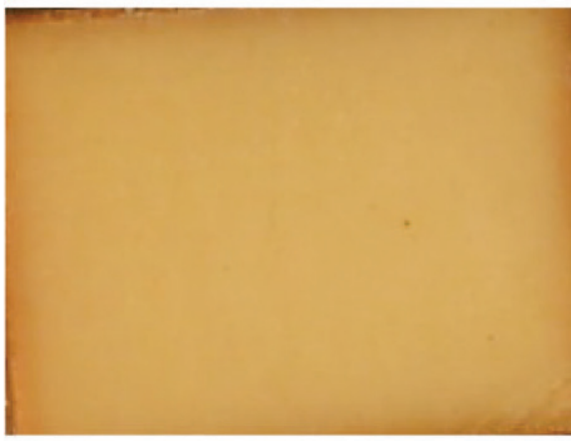

D

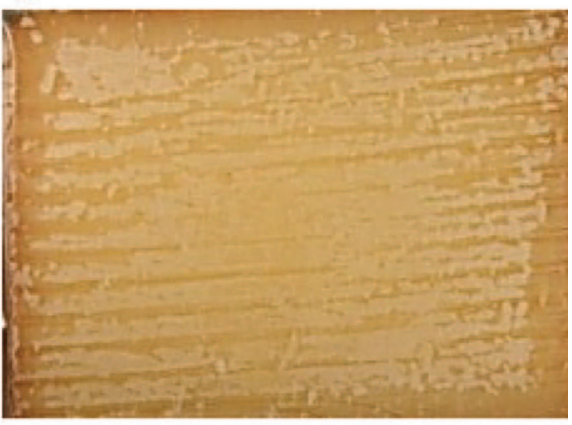

Figure 4. Digital images of calcium lactate crystals on the surface of smoked Cheddar cheese slices that were vacuum packaged and stored for $20 \mathrm{wk}$ at $1^{\circ} \mathrm{C}$ as follows: (A) very tight packaging, smooth surface; (B) very tight packaging, rough surface; (C) moderately tight packaging, smooth surface; and (D) moderately tight packaging, rough surface.

A

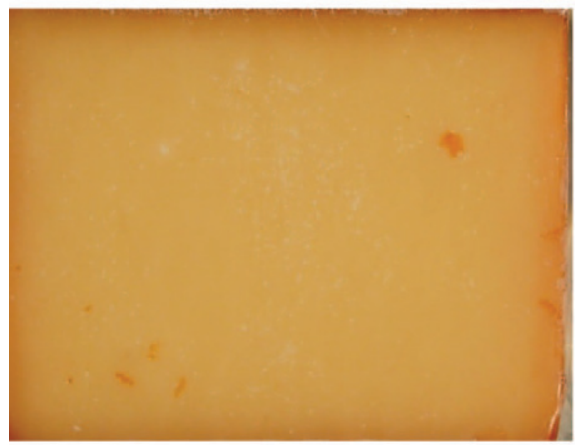

C

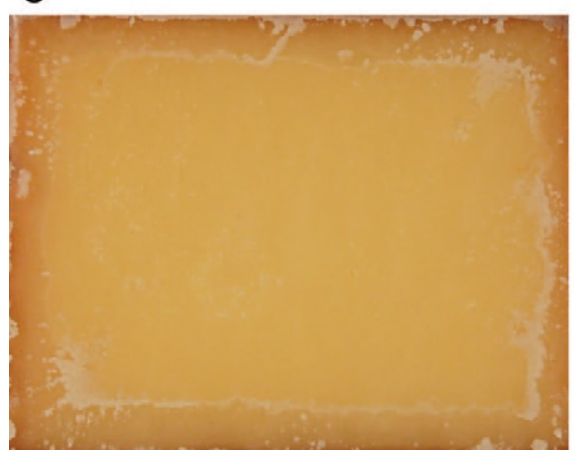

B

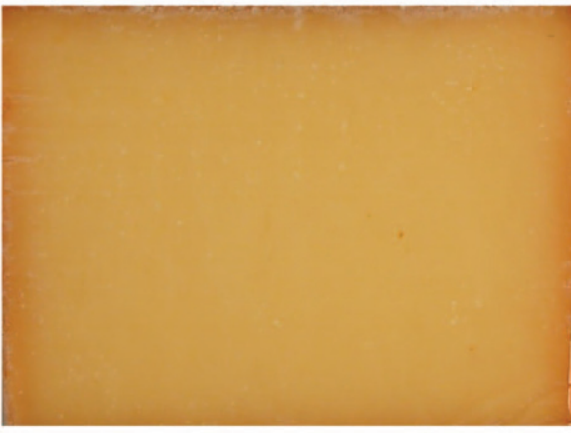

D

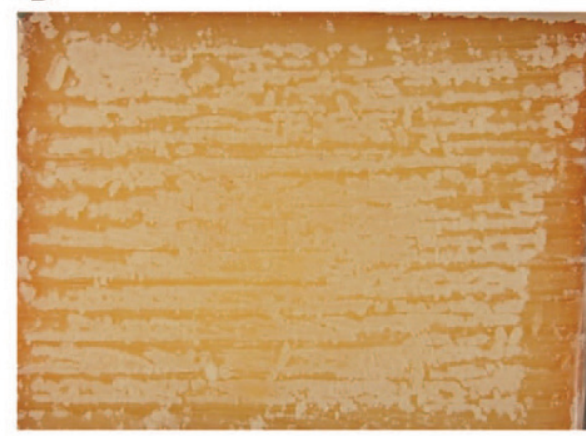

Figure 5. Digital images of calcium lactate crystals on the surface of smoked Cheddar cheese slices that were stored for an additional 12 mo at $1^{\circ} \mathrm{C}$ following completion of the 20 -wk study as follows: (A) very tight packaging, smooth surface; (B) very tight packaging, rough surface; (C) moderately tight packaging, smooth surface; and (D) moderately tight packaging, rough surface. 
force of the packaging film on the cheese surface that occurred when the vacuum was pulled to $1 \mathrm{kPa}$ before heat-sealing the film (i.e., the conditions used for the very tight packaging treatment) was great enough to compress the surface and smooth out irregularities, even with surfaces that had been deliberately etched with a cheese grater to create rough irregularities (Figure 4B).

In contrast, the smooth surface of the slice that was packaged moderately tightly (Figure 4C) developed crystals along the periphery of the slice because the packaging film adhered less tightly around the edges, thus creating an environment for nucleation to occur. The opposite (i.e., rough) surface of the same slice packaged moderately tightly, shown in Figure 4D, displayed extensive crystallization that was arrayed along the rough ridges that lined the grooves that were etched into the cheese surface using a cheese grater. Thus, the downward force of the packaging film on the cheese surface that occurred when the vacuum was pulled only to $10 \mathrm{kPa}$ before heat-sealing the film (i.e., moderately tight packaging) was not sufficient to compress the surface and smooth out the irregularities that had been deliberately etched into the surface. The irregular surface, in turn, presented an environment that was highly favorable to crystal nucleation and subsequent growth to a visible threshold. This was in striking contrast to the rough surfaces that were packaged very tightly (Figure 4B), which displayed no rough irregularities because the pressure of the packaging film compressed the cheese surface to a uniformly smooth topography, which strongly suppressed crystal nucleation.

Results of this study indicate that very tight $(1 \mathrm{kPa})$ packaging in general and the combination of smooth surface plus very tight packaging in particular were remarkably effective in combating surface crystallization by eliminating potential sites for crystal nucleation. Indeed, tight packaging continued to inhibit crystallization long after completion of the 20-wk study, as illustrated in Figure 5, which compares images of the same surfaces shown in Figure 4 after 1 yr of additional storage at $1^{\circ} \mathrm{C}$. The combination of rough surface and moderately tight packaging continued to display extensive white crystallization along the rough ridges lining the grooves that were etched into cheese surface (Figure 5D). The opposite smooth surface displayed heavy crystallization along the edges where the packaging film adhered less tightly to the cheese surface, but not on the center of the slice where the film remained totally in contact with the cheese surface (Figure 5C). In contrast, slices with both rough (Figure 5B) and smooth (Figure 5A) surfaces that were packaged very tightly displayed only tiny crystals that were almost imperceptible to the naked eye.

\section{CONCLUSIONS}

Loose packaging (i.e., absence of contact between the cheese surface and packaging film) strongly promoted CL crystal formation, irrespective of surface roughness. Rough cheese surface combined with moderately tight packaging (i.e., almost complete contact between the cheese surface and packaging film but little pressure exerted by the film on the cheese surface, which allowed surface irregularities to remain intact) also established a surface environment that was favorable for CL crystal nucleation and growth. In contrast, crystal nucleation and growth were strongly inhibited when rough cheese surface was combined with very tight packaging, evidently because the strong pressure exerted by the packaging film compressed the cheese surface and smoothed out the irregularities. Finally, a smooth cheese surface combined with very tight packaging presented the greatest barrier to surface crystallization. Our results indicate that the number and distribution of nucleation sites at the cheese surface influence the crystallization behavior of smoked Cheddar cheese that is saturated with respect to calcium lactate and thus predisposed to crystal formation. Furthermore, packaging tightness and cheese surface roughness appeared to act synergistically to modulate the number of nucleation sites at the cheese surface and the rate of visible crystal formation. Therefore, optimizing the cut-and-wrap operation to limit the number of surface nucleation sites by minimizing surface roughness and maximizing package tightness may offer a strategy to slow down or inhibit CL crystal formation in smoked Cheddar cheeses that are predisposed to crystallize. Surface roughness and packaging tightness may have similar modulating effect on surface nucleation sites and rate of crystallization in Cheddar cheese that is not smoked, but which is saturated with respect to calcium lactate; however, additional work will be needed to demonstrate that these effects also apply to unsmoked Cheddar.

\section{ACKNOWLEDGMENTS}

The financial support of the New England Dairy Promotion Board (Winooski, VT), Vermont Dairy Promotion Council (Montpelier), and USDA Hatch Project VT-HO1305 are gratefully acknowledged. The technical assistance of Christine Ogg Nelson (Fletcher Allen Health Care, Burlington, VT) during sample preparation and photographing is gratefully acknowledged.

\section{REFERENCES}

Agarwal, S., M. Costello, and S. Clark. 2005. Gas-flushed packaging contributes to calcium lactate crystals in Cheddar cheese. J. Dairy Sci. $88: 3773-3783$. 
Agarwal, S., J. R. Powers, B. G. Swanson, S. Chen, and S. Clark. 2006a. Cheese $\mathrm{pH}$, protein concentration, and formation of calcium lactate crystals. J. Dairy Sci. 89:4144-4155.

Agarwal, S., J. R. Powers, B. G. Swanson, S. Chen, and S. Clark. 2008 Influence of salt-to-moisture ratio on starter culture and calcium lactate crystal formation. J. Dairy Sci. 91:2967-2980.

Agarwal, S., K. Sharma, B. G. Swanson, G. Ü. Yüksel, and S. Clark. 2006b. Nonstarter lactic acid bacteria biofilms and calcium lactate crystals in Cheddar cheese. J. Dairy Sci. 89:1452-1466.

Chou, Y. E., C. G. Edwards, L. O. Luedecke, M. P. Bates, and S. Clark. 2003. Nonstarter lactic acid bacteria and aging temperature affect calcium lactate crystallization in Cheddar cheese. J. Dairy Sci. 86:2516-2524

Dybing, S. T., J. A. Wiegand, S. A. Brudvig, E. A. Huang, and R. C. Chandan. 1988. Effect of processing variables on the formation of calcium lactate crystals on Cheddar cheese. J. Dairy Sci. $71: 1701-1710$

Hartel, P. 2001. Crystallization in Foods. Aspen Publishers, Gaithersburg, MD.

IDF (International Dairy Federation). 1993. Determination of the nitrogen content. Provisional IDF Int. Standard 20B:1993:Part 3. IDF, Brussels, Belgium.

Johnson, M. 2004. Revisiting calcium lactate crystals in cheese. Dairy Pipeline 16:1-11.

Johnson, M. E., B. A. Riesterer, C. Chen, B. Tricomi, and N. F. Olson. 1990a. Effect of packaging and storage conditions on calcium lactate crystallization on the surface of Cheddar cheese. J. Dairy Sci. 73:3033-3041.

Johnson, M. E., B. A. Riesterer, and N. F. Olson. 1990b. Influence of nonstarter bacteria on calcium lactate crystallization on the surface of Cheddar cheese. J. Dairy Sci. 73:1145-1149.

Marshall, R. T. 1993. Standard Methods for the Examination of Dairy Products. 16th ed. American Public Health Association, Washington, DC.
Rajbhandari, P., and P. S. Kindstedt. 2005a. Compositional factors associated with calcium lactate crystallization in smoked Cheddar cheese. J. Dairy Sci. 88:3737-3744.

Rajbhandari, P., and P. S. Kindstedt. 2005b. Development and application of image analysis to quantify calcium lactate crystals on the surface of smoked Cheddar cheese. J. Dairy Sci. 88:4157-4164.

Rajbhandari, P., and P. S. Kindstedt. 2008. Characterization of calcium lactate crystals on Cheddar cheese by image analysis. J. Dairy Sci. 91:2190-2195.

Rajbhandari, P., J. Patel, E. Valentine, and P. S. Kindstedt. 2009. Chemical changes that predispose smoked Cheddar cheese to calcium lactate crystallization. J. Dairy Sci. 92:3616-3622.

Rajbhandari, P.. J. Patel, E. Valentine, and P. S. Kindstedt. 2013. Effect of storage temperature on crystal formation rate and growth rate of calcium lactate crystals on smoked Cheddar cheeses. J. Dairy Sci. 96:3442-3448.

Somers, E. B., M. E. Johnson, and A. C. L. Wong. 2001. Biofilm formation and contamination of cheese by nonstarter lactic acid bacteria in the dairy environment. J. Dairy Sci. 84:1926-1936.

Swearingen, P. A., D. E. Adams, and T. L. Lensmire. 2004. Factors affecting calcium lactate and liquid expulsion defects in Cheddar cheese. J. Dairy Sci. 87:574-582.

Valentine, E., P. Rajbhandari, J. Patel, and P. S. Kindstedt. 2007. Nucleation and growth rates of calcium lactate crystals on smoked Cheddar cheese. 2. Effect of packaging tightness. J. Dairy Sci. 90(Suppl. 1):198. (Abstr.)

Zanotto, E. D., and V. M. Fokin. 2003. Nucleation control. Pages 591-613 in Philosophical Transactions: Mathematical, Physical and Engineering Sciences, Vol. 361, No. 1804. The Royal Society, London, UK. 